\title{
ANALYSIS OF FINITE ELEMENT SOLUTION OF THE POISSON-BOLTZMANN EQUATION
}

\author{
N. S. V. BARBOSA ${ }^{1}$, E. R. A. LIMA ${ }^{1}$ e F. W. TAVARES ${ }^{2,3}$ \\ ${ }^{1}$ Universidade do Estado do Rio de Janeiro, Programa de Pós-graduação em Engenharia \\ Química \\ ${ }^{2}$ Universidade Federal do Rio de Janeiro, Escola de Química \\ ${ }^{3}$ Universidade Federal do Rio de Janeiro, PEQ/COPPE \\ E-mail address: nathalia.vernin@gmail.com
}

\begin{abstract}
The Poisson-Boltzmann equation (PBE) is a second-order partial differential equation that can be used to describe the ion distribution of colloidal systems. In its classical form, only the electrostatic interactions between ions and interface are considered. The aim of this work is to study the solution of PBE by second order spline finite elements method applied to a one-dimensional system. This method is used to solve the classical and the modified PB equation, which considers van der Waals dispersion (non-electrostatic) interactions between ions and macro-particles. The method consists in dividing of the domain into a number of subdomains, in which the dependent variable is approximated by a second order polynomial. The algorithm developed is validated by comparing the numerical results of the classical PBE with results from the literature.
\end{abstract}

\section{INTRODUCTION}

Many techniques can be used to model colloidal system, such as: implicit and explicit methods. Implicit methods consider the solvent as a continuous medium whose characteristics are described by a single parameter: the dielectric constant. These methods reduce the degrees of freedom of the system. Examples of implicit models are: PBE (ALIJÓ, 2011; BAKER, 2005; LIMA et al., 2009; MOREIRA, 2007), Coulomb's law and generalized Born methods (FEIG; BROOKS, 2004).

Poisson-Boltzmann equation (PBE - Equation 1) is a second-order elliptic partial differential equation. In the classical form, it is obtained from the Poisson equation assuming that the mobile charges follow a Boltzmann distribution in the mean field approximation. This distribution is affected by electrostatic potential and temperature, following the GouyChapman approach to describe the electrical double layer.

$$
\varepsilon_{0} \nabla \cdot(\varepsilon \nabla \psi)=-e \sum_{i} z_{i} c_{i, \infty} \exp \left(\frac{-e z_{i} \psi}{k_{B} T}\right)-\rho_{f}
$$

where $\varepsilon_{0}$ is the permittivity of vacuum, $\varepsilon$ is the dielectric constant, $c_{i, \infty}$ is the concentration of ion $i$ (number of ions per volume unit) at an infinitely large distance from the solid phase (in the bulk solution, $\psi_{\infty}=0$ ), $e$ is the elementary charge, $z_{i}$ is the charge number of ion $i, \psi$ is the electrostatic potential, $k_{B}$ is the Boltzmann constant, $T$ is the absolute temperature of 
the system and $\rho_{f}$ is the volumetric density of fixed charge.

In the classical theory, the PBE takes into account only the electrostatic potential. But it is not able to predict the ion specificity commonly observed experimentally. Aiming at the inclusion of other interactions beyond the electrostatic, we use an ion specific PBE (ALIJÓ, 2011; BARBOSA, 2014; LIMA, 2008; MOREIRA, 2007):

$$
\varepsilon_{0} \nabla \cdot(\varepsilon \nabla \psi)=-e \sum_{i} z_{i} c_{i, \infty} \exp \left(-\frac{z_{i} e \psi+\sum_{j} U_{i, j}}{k_{B} T}\right)-\rho_{f}
$$

where $U_{i, j}$ is the $j$-th non-electrostatic energetic contribution of ion $i$. Non-electrostatic interactions can be, for example, van der Waals interactions between ion $i$ and the interface, and hydration interactions. Moreira (2007) studied the influence of the dispersion potential between ions and macro-particles on different colloid systems, showing that the inclusion of this potential is relevant to explain some phenomena such as adsorption of colloidal particles and surface tension of electrolyte solutions.

Note that if non-electrostatic interactions $U_{i, j}$ are disregarded, Equation (2) reduces to Equation (1). Thus, PBE in its classical form is a particular case of the modified PBE.

For numerical convenience, we write Equation (2) in dimensionless form as:

$$
\nabla \cdot(\bar{\varepsilon} \nabla y)=-\frac{\sum_{i} z_{i} c_{i, \infty} \exp \left(-z_{i} y-\sum_{j} u_{i, j}\right)}{\sum_{i} z_{i}^{2} c_{i, \infty}}-\bar{\rho}_{f}
$$

where $y=e \psi / k_{B} T$ is the dimensionless electrostatic potential, $\bar{\varepsilon}$ is the normalized dielectric constant, that is equal to $\bar{\varepsilon}=\varepsilon / \varepsilon_{W}, \varepsilon_{W}$ is the dielectric constant of water at system temperature, $u_{i, j}=\sum_{i} U_{i, j} / k_{B} T$ are the dimensionless non-electrostatic potentials and $\bar{\rho}_{f}$ is the dimensionless volumetric density of fixed charges.

In Equation (3), all spatial variables are scaled by $\kappa^{-1}$, the Debye length:

$$
\kappa^{2}=\frac{e^{2} \sum_{i} c_{i, \infty} z_{i}^{2}}{\varepsilon_{W} \varepsilon_{0} k_{B} T}
$$

In this work, we consider only the van der Waals dispersion interactions obtained by Lifshitz's theory to calculate ion specific non-electrostatic interactions between ions and interface (LIMA, 2008; TAVARES et al., 2004). In Cartesian unidirectional coordinates, these interactions are given by (ALIJÓ, 2011; LIMA, 2008; MOREIRA, 2007; NINHAM; LO NOSTRO, 2010):

$$
U_{i}=-\frac{B_{i}}{x^{3}}, \quad \text { to } x \geq r_{i}
$$

where $x$ is the perpendicular distance from the center of the ion to the interface. According to Ninham and Lo Nostro (2010), this expression is valid from a distance between the center of the ion and the surface equivalent to one or two times the ion diameter. When $B_{i}$ coefficient is 
positive, interaction is attractive, promoting, in general, ion adsorption at the interface. On the other hand, a negative $B_{i}$ value indicates repulsive interaction, promoting, in general, ion depletion of the interface.

The aim of this work is to study the solution of dimensionless PBE by spline collocation in finite elements in a one-dimensional system in Cartesian and spherical coordinates.

Despite having limitations for divalent ions or phenomena in which the correlation between the ions is important, PBE has the advantage of being solved with low computational cost and having no numerical instability for highly asymmetric systems, as happens with integral equations theories (LIMA et al., 2009).

\section{THEORY}

\subsection{Finite element method}

The finite element method consists in the division of the domain, which defines the problem that we want to model into smaller subdomains. In each of these subdivisions, the dependent variables involved in the problem are approximated by interpolating its values fixed in the boundaries of the subdomain. Thus, the discretization of the differential equation results in algebraic equations whose unknowns are the values of these variables on the boundary of various subdivisions (nodes of the finite elements) (PINTO; LAGE, 2001; DHATT et al., 2012).

As an example for application of spline collocation in finite elements method for steady one-dimensional system, we consider the general equation:

$$
\frac{d}{d x}\left(\Theta \frac{d y}{d x}\right)+S=0
$$

where $x$ is the independent variable, $y$ is the dependent variable and $S$ is the source term.

In the development of the second order spline collocation in finite elements method, a second order polynomial is used as interpolating function inside each subdomain. In order to solve the problem it is necessary to find the coefficients of the polynomial inside each subinterval (finite element) (PINTO, LAGE, 2001). Thus, we have:

$$
\begin{aligned}
& y(x, a, b, c)=a+b x+c x^{2} \\
& y^{\prime}(x, b, c)=b+2 c x \\
& y^{\prime \prime}(x, c)=2 c
\end{aligned}
$$

For each $i$ element, varying from 1 to $n$ (number of finite elements), there is a set of second order spline coefficients $\left(a_{i}, b_{i}\right.$, and $\left.c_{i}\right)$.

Residue is the function obtained when the approach proposed is replaced into the 
differential equation to be solved. Thus, if the approximation is exact, the residue is zero in the points of the considered interval.

If we divide the interval into $n$ finite elements, at which $x m_{i}$ is the boundary of each element $i$ (ranging from 1 to $n-1$ ) and using second order polynomial approximations, there are $3 n$ parameters. To solve this system in order to obtain a unique solution, it is necessary $3 n$ equations. The residual system for a second order differential equation is composed of boundary conditions substituted by polynomial approximation, continuity conditions of the spline at elements' boundary, differentiability conditions of the spline at elements' boundary and differential equations replaced with polynomial approximation at collocation points.

Two equations are obtained from boundary conditions. Considering Dirichlet boundary conditions (dependent variable specified at the boundary) we have the first two algebraic equations of the residual system:

$$
\begin{aligned}
& \operatorname{res}_{1}=y\left(x_{0}, a_{1}, b_{1}, c_{1}\right)-\left.y\right|_{x=x_{0}} \\
& \operatorname{res}_{2}=y\left(x_{f}, a_{n}, b_{n}, c_{n}\right)-\left.y\right|_{x=x_{f}}
\end{aligned}
$$

For Neumann boundary conditions (derivatives specified the boundary) we have:

$$
\begin{aligned}
& \operatorname{res}_{1}=y^{\prime}\left(x_{0}, b_{1}, c_{1}\right)-\left.y^{\prime}\right|_{x=x_{0}}=0 \\
& \operatorname{res}_{2}=y^{\prime}\left(x_{f}, b_{n}, c_{n}\right)-\left.y^{\prime}\right|_{x=x_{f}}=0
\end{aligned}
$$

Regarding the continuity conditions of the spline at elements' boundary, there are $n-1$ equations. For $i$ ranging from 1 to $n-1$, we have:

$$
\operatorname{res}_{2+i}=y\left(x m_{i}, a_{i}, b_{i}, c_{i}\right)-y\left(x m_{i+1}, a_{i+1}, b_{i+1}, c_{i+1}\right)=0
$$

For the differentiability conditions of the spline at elements' boundary, there are $n-1$ equations.

$$
\operatorname{res}_{1+n+i}=y^{\prime}\left(x m_{i}, b_{i}, c_{i}\right)-y^{\prime}\left(x m_{i+1}, b_{i+1}, c_{i+1}\right)=0
$$

Replacing the polynomial approximation in the differential equation at collocation points inside each subinterval, we obtain $n$ equations of the residual system. Here the collocation points $\left(x c_{i}\right)$ are defined as the midpoint of each element. In this way, there are $n$ collocation points.

When the source term is nonlinear, the algebraic system to be solved is also nonlinear. A strategy used to solve the problem is the linearization of the source term around the previous iteration value of $y$ at the collocation point of finite element $\left(y_{c}\right)$.

$$
S=S_{c} y_{c}+S_{P}
$$

where $S_{c}$ and $S_{P}$ are the coefficients obtained from the values of $y_{c}$ in the previous iteration. Thus, the solution should be iterative, i.e., the linearized equations are solved at each iteration 
and, therefore, the solution of nonlinear equations are obtained after the convergence of consecutive iterations (LIMA, 2008; PINTO, LAGE, 2001). It is noteworthy that the linear system obtained is a sparse system. As a strategy for resolution, we chose to order the equations to obtain a band system composed of main diagonal and three diagonals immediately above this and two diagonals immediately below the main diagonal. The method used to solve the linear algebraic system is a generalized Thomas method for solving heptadiagonal systems (BARBOSA, 2014).

\subsection{Applying finite element method to the Poisson-Boltzmann Equation}

Considering the dimensionless PBE, Equation (3(3), and comparing it with Equation (6), we can identify the corresponding $S$ and $\Theta$ terms. Thus, classical and modified PBE can be solved for different coordinates applying finite element method just by changing the coefficients and source term. Table 1 shows the values of $S$ and $\Theta$ that should be used for Cartesian and spherical coordinates, both in one-dimensional. While solving the nonlinear algebraic system, the iterative linearization of the $S$ term around a $y_{c}$ point of the previous iteration $\left(y_{c}^{*}\right)$ results in Equation (16). In this case $S_{c}$ and $S_{P}$ coefficients can be obtained from the first two terms of the Taylor series of the PBE's right side around $y_{c}^{*}$.

Table 1- Poisson-Boltzmann terms for different one-dimensional coordinates

\begin{tabular}{|cccc|} 
Coordinates & $\begin{array}{c}\text { Independent } \\
\text { Variable }\end{array}$ & $\boldsymbol{\Theta}$ & $\boldsymbol{S}$ \\
\hline Cartesian & $x$ & $\bar{\varepsilon}$ & $\frac{\sum_{i} z_{i} c_{i, \infty} \exp \left(-z_{i} y-u_{i}\right)}{\sum_{i} z_{i}{ }^{2} c_{i, \infty}}-\bar{\rho}_{f}$ \\
\hline Spherical & $r$ & $\bar{\varepsilon} r^{2}$ & $r^{2}\left(\frac{\sum_{i} z_{i} c_{i, \infty} \exp \left(-z_{i} y-u_{i}\right)}{\sum_{i} z_{i}{ }^{2} c_{i, \infty}}\right)-\bar{\rho}_{f}$ \\
\hline
\end{tabular}

\section{RESULTS}

In order to validate the implementation of the numerical method, the numerical results were compared to the analytical solution obtained from the Gouy-Chapman approach in Cartesian coordinates, without including the non-electrostatic potential (MOREIRA, 2007; LIMA, 2008; BARBOSA, 2014). This is a classical one-dimensional problem for a single plate with specified surface potential equal to $-15 \mathrm{mV}$ in an aqueous system. The system is composed of a symmetric electrolyte $z: z$ at a bulk concentration equal to $150.0 \mathrm{mM}$ and temperature equal to $298.15 \mathrm{~K}$. The dielectric constant of the medium corresponds to that for water at $298.15 \mathrm{~K}(\varepsilon=78.5)$.

In spherical coordinates, when the radius of a charged particle is large enough, the solution tends to approach to the solution in Cartesian coordinates. Thus, we compared solutions for spherical coordinates considering three different particle radiuses under the same conditions. According to Figure 1B the solution for charged particles with radius of the order of $10^{-7} \mathrm{~m}$ or higher agrees with the analytical solution in Cartesian coordinates. Figure $1 \mathrm{~A}$ 
shows that the numerical solution is satisfactory when compared with analytical solution.
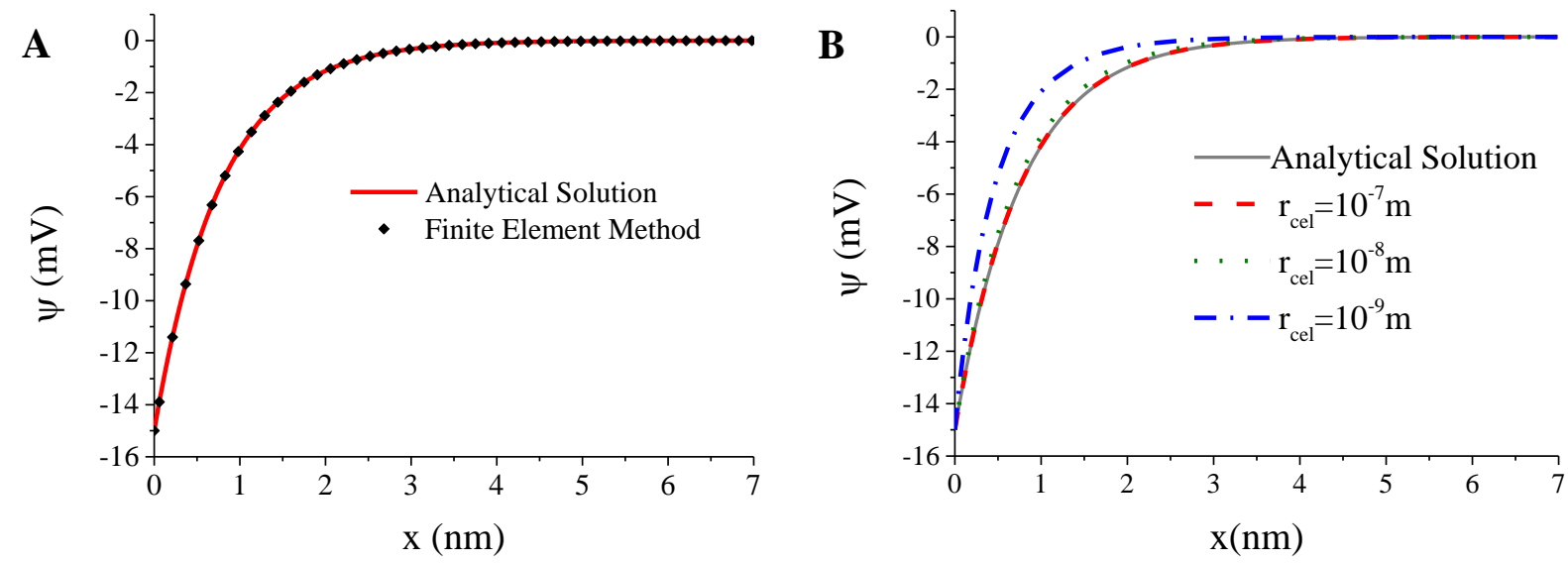

Figure 1 - Validation of the spline collocation method in Cartesian coordinates (A) and comparison of numerical spline collocation solution in spherical coordinates with the analytical solution in Cartesian coordinates (B)

The third case to be analyzed is the modified PBE with the inclusion of van der Waals interactions between ions and colloid via Lifshitz theory. The potential at a specified distance from $2 \AA$ (distance equivalent to the ionic radius) is equal to $-15 \mathrm{mV}$. $\mathrm{NaCl}$ and $\mathrm{NaI}$ electrolytes are present in equimolar concentrations in the bulk solution. Dispersion interactions coefficients between the surface and the ions $\mathrm{Na}^{+}, \mathrm{Cl}^{-}$and $\mathrm{I}^{-}$are: $0.454 \times 10^{-50}$ $\mathrm{Jm}^{3}, 3.576 \times 10^{-50} \mathrm{Jm}^{3}$ and $5.712 \times 10^{-50} \mathrm{Jm}^{3}$, respectively.

In Figure 2 we show the relative ionic concentration profile (ratio between the ion concentration in $x$ and its bulk concentration). Note that near the surface there is a higher concentration of the ion $\mathrm{I}^{-}$due to its higher polarizability compared to $\mathrm{Cl}^{-}$. The more polarizable the ion, the greater it tends to adsorb on the surface.

Boström et al. (2009) obtained similar results when analyzing ionic partition between two regions: a neutral colloidal particles (micelles) solution and a micelle free phase. They observed that the more polarizable the ion, the more it tends to accumulate in the dense phase, adsorbing on the micelle surfaces.

Figure 3 shows the ionic concentration profile of a system with an uncharged membrane at $1.0 \mu \mathrm{m}$ with a thickness of $10 \mathrm{~nm}$. The phase at left of the membrane has a constant volumetric density of fixed charges equal to $-9,64 \mathrm{MC} / \mathrm{m}^{3}$. To define the volumetric density of fixed charge in both sides of the membrane, we use a regularization function, according with Freitas (2012) and Barbosa (2014). The bulk concentrations of the salts are: $60 \mathrm{mM} \mathrm{NaCl}$ and $90 \mathrm{mM}$ NaI. The dispersion interactions coefficients between the surface and the ions $\mathrm{Na}^{+}, \mathrm{Cl}^{-}$ and $\mathrm{I}^{-}$are the same of the previous case. It is noteworthy that the ionic concentration profile inside the membrane is not real, being used only for numerical convenience of ensuring the continuity of the system. 


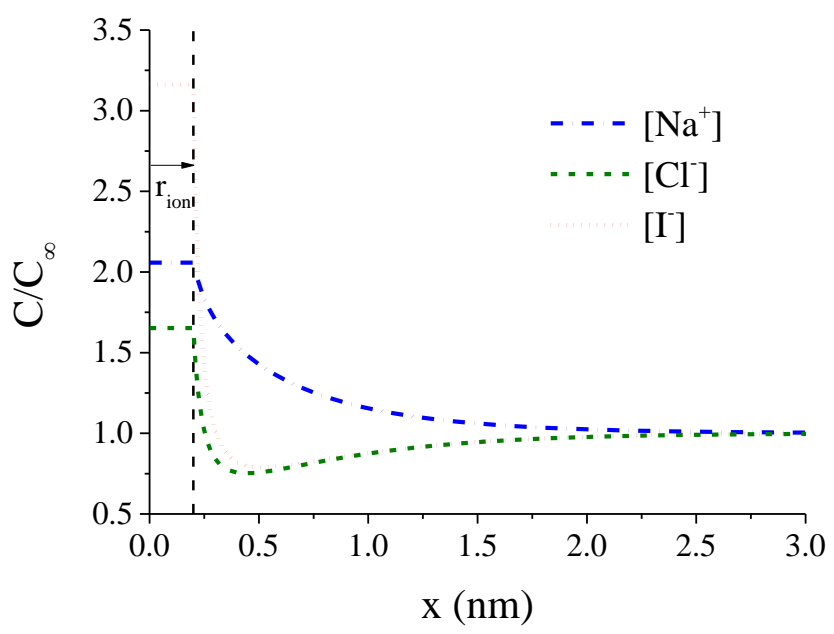

Figure 2 - Relative ionic concentration profile near a charged surface for $\psi(x=2 \AA)=$ $-15,0 \mathrm{mV}$ at $298.15 \mathrm{~K}$. The plateau at distance of $2 \AA$ corresponds to the ionic radius.

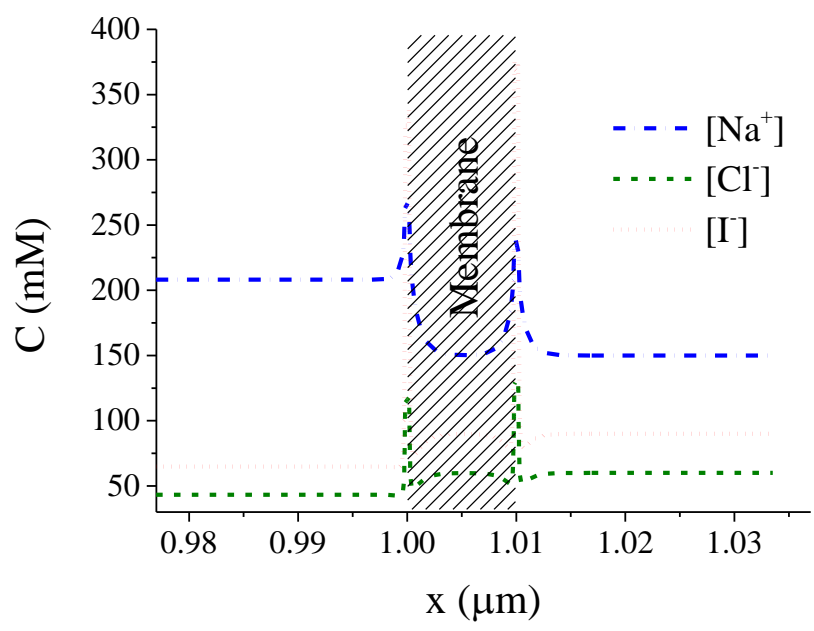

Figure 3 - Ionic concentration profile of a system with an uncharged membrane at $1.0 \mu \mathrm{m}$ with a thickness of $10 \mathrm{~nm}$ (shaded region of the graph). The bulk concentrations are $60 \mathrm{mM}$ $\mathrm{NaCl}$ and $90 \mathrm{mM} \mathrm{NaI}$.

\section{CONCLUSIONS}

We have implemented the second order spline finite elements method to solve the classical and modified PBE in Cartesian and spherical coordinates. The method was validated by comparison with the analytical solution of classical PBE in Cartesian coordinates. Thus, it has been proved to be efficient and suitable method for Poisson-Boltzmann calculations. We have also considered a system with fixed charges and non-electrostatic interactions between ions and macro-particles (e.g. membrane and proteins). The successful application of the numerical method to this stiff system proves its robustness and shows that it may be useful to study the ionic partition in biological system. 


\section{REFERENCES}

ALIJÓ, P. H. R. Cálculo de propriedades físico-químicas de sistemas coloidais assimétricos via equação de Poisson-Boltzmann modificada. 2011. 112 f. Dissertação (Mestrado em Engenharia Química) - Programa em Pós-Graduação em Engenharia Química, COPPE, Universidade Federal do Rio de Janeiro, Rio de Janeiro, 2011.

BARBOSA, N. S. V. Aplicação da equação de Poisson-Boltzmann modificada em sistemas biológicos: análise da partição iônica em um eritrócito. 2014. 149 f. Dissertação (Mestrado em Engenharia Química) - Programa de Pós-graduação em Engenharia Química, Universidade do Estado do Rio de Janeiro, Rio de Janeiro, 2014.

BAKER, N. A. Improving implicit solvent simulations: a Poisson-centric view. Curr. Opin. Struct. Biol., v. 15, n. 2, p. 137-143, 2005.

BOSTRÖM, M.; LIMA, E. R. A.; BISCAIA, E. C.; TAVARES, F. W.; LO NOSTRO, P.; PARSONS, D. F.; DENIZ, V.; NINHAM, B. W. Anion-specific partitioning in twophase finite volume systems: possible implications for mechanisms of ion pumps. $J$. Phys. Chem. B, v. 113, n. 23, p. 8124-8127, 2009.

DHATT, G.; LEFRANÇOIS, E.; TOUZOT, G. Finite element method. Hoboken: John Wiley \& Sons and ISTE, 2012.

FEIG, M.; BROOKS, C. L. I. Recent advances in the development and application of implicit solvent models in biomolecule simulations. Curr. Opin. Struct. Biol., v. 14, n. 2, p. $217-$ 224, 2004.

FREITAS, T. C.; QUINTO, T. C.; SECCHI, A. R.; BISCAIA, E. C. An Efficient adjoint-free dynamic optimization methodology for batch processing using Pontryagin's formulation. In: BOGLE, I. D. L.; FAIRWEATHER, M. (Ed.) 22nd European Symposium on Computer Aided Process Engineering. Amsterdam: Elsevier, 2012.

LIMA, E. R. A.; BISCAIA, E. C.; TAVARES, F. W. Modelagem computacional de sistemas coloidais. In: NETO, M. F.; PLATT, G.; BASTOS, I.; ROCHA, M.; HENDESON, N. Modelagem computacional em materiais. Rio de Janeiro: Ciência Moderna, 2009.

MOREIRA, L. A. Cálculo de propriedades físico-químicas de sistemas coloidais via equação de Poisson-Boltzmann: efeito da inclusão de potenciais não-eletrostáticos. $2007.127 \mathrm{f}$. Dissertação (Mestrado em Ciência da Tecnologia de Processos Químicos e Bioquímicos) - Programa em Pós-Graduação em Tecnologia de Processos Químicos e Bioquímicos, Escola de Química, Universidade Federal do Rio de Janeiro, Rio de Janeiro, 2007.

NINHAM, B. W. On progress in forces since the DLVO theory. Adv. Colloid Interface Sci., v. 83, n. 1-3, p. 1-17, 1999.

NINHAM, B. W.; LO NOSTRO, P. Molecular Forces and Self Assembly. Cambridge: Cambridge University Press, 2010.

PINTO, J. C.; LAGE, P. L. C. Métodos numéricos em problemas de engenharia química. Série escola piloto em engenharia química, COPPE/UFRJ. Rio de Janeiro: e-papers, 2001.

TAVARES, F. W.; BRATKO, D.; BLANCH, H. W.; PRAUSNITZ, J. M. Ion-specific effects in the colloid-colloid or protein of mean force: role of salt-macroion van der Waals interactions. J. Phys. Chem. B, v. 108, n. 26, p. 9228-9235, 2004. 